\title{
Ancora un plagio in Italia del Tirant lo Blanc: la lettera dedicatoria alla Tragicocomedia di Calisto e Melibea (1506)
}

\section{An other plagiarism of Tirant lo Blanc in Italy: the dedicatory letter of Tragicocomedia di Calisto e Melibea (1506)}

JACOPO GESIOT

gesiot.jacopo@spes.uniud.it

ORCID 0000-0003-2969-5515

Università di Udine

Riassunto: Nel 1506 usciva a Roma la prima versione italiana de La Celestina, corredata da una lettera dedicatoria del traduttore in gran parte risultato del plagio di diversi passaggi del Tirant lo Blanc di Joanot Martorell. Il presente articolo ha lo scopo di rendere notizia di questo stratagemma e, soprattutto, di chiarire le ragioni della contaminazione: all'inizio del Cinquecento la lettura del Tirant, oltre a essere un'occasione di svago, preservava il suo motivo formativo, come dimostrato dall'intento didascalico (almeno apparente) dichiarato dal primo traduttore certo di un brano di Martorell: Alfonso Ordóñez.

Parole chiave: Tirant lo Blanc, traduzione, La Celestina, Rinascimento italiano.

Abstract: The first translation of La Celestina appears in Rome in 1506 and it was furnished with a dedicatory letter by the translator, mostly composed of plagiarized fragment from Tirant lo Blanc by Joanot Martorell. The present article aims to publicize this stratagem and especially to explain the reason of this fusion: in the early of 15 th century the reading of Tirant, besides the amusement, preserves its educational purpose, as we can see from the didactic intent of the first translator of some excerpts from Martorell: Alfonso Ordóñez.

Keywords: Tirant lo Blanc, translation, La Celestina, Italian renaissance. *Il mio ringraziamento e il mio omaggio vanno a Josep Pujol Gómez, che, nel rivedere la mia tesi di dottorato, ha individuato per
primo questo tema e me ne ha fatto dono. 
Molto si è scritto a proposito della relazione -forse pure della derivazione- che lega romanzo cavalleresco del medio Quattrocento di Joanot Martorell e La Celestina, in particolare, come ha più volte chiarito la critica, per ciò che riguarda la parabola dei protagonisti maschili Tirant e Calisto. ${ }^{1}$ Tra i temi ricorrenti, al di là dei riferimenti anche puntuali che coinvolgono altri personaggi e altre dinamiche, possiamo ricordare qui, in termini generali, la confusione delle gerarchie sociali che anima lo sforzo dei due amatori; l'eccesso cortese e la convulsione che contraddistinguono la loro strategia; infine, il verdetto tragico e comico, nel senso di humilior, che corona la loro impresa. Invero, tutti sintomi e segni di un mutamento concreto che le élite del tardo Medioevo e della prima età moderna, visto il normale ritardo nel trapasso dei concetti, non potevano che interpretare nei termini dello scadimento ideale. ${ }^{2}$ Quindi, senza riferire i dettagli testuali di questa connessione, ci si è appena limitati a delineare una meccanica comune, anche piuttosto banale, che tornerà utile nel seguito dell'articolo, in modo da stabilire un raccordo con l'immaginazione dei lettori italiani di inizio Cinquecento.

È infatti tra il 1505 e il 1506 che l'altrimenti oscuro traduttore Alfonso Ordóñez sceglie di indirizzare la sua Tragicocomedia di Calisto e Melibea (Roma, Eucario Silber, 1506) a «madonna Gentile Feltria de Campo Fregoso», mostrando nella sua dedicatoria di avere ben presente il Tirant lo Blanc, uscito quindici anni prima a Valencia (Nicolaus Spindeler, 1490) e inseguito a Barcellona nel 1497. ${ }^{3}$ Poco sappiamo di questo spagnolo, forse giunto in Italia per attrazione della corte iberica di Alessandro VI e poi entrato nel seguito di Giulio II, un papa che, nonostante l'antipatia per il Borgia, lasciava spazio all'osmosi culturale italo-spagnola inaugurata dal predecessore. ${ }^{4}$ A questo proposito, mentre pare dubbia la notizia di una rappresentazione romana de La Celestina già in occasione del matrimonio tra Alfonso I d'Este e Lucrezia Borgia, è invece probabile che il rapporto tra lo spagnolo e Gentile da Campofregoso dipendesse dalla prossimità al Della Rovere, dal momento che il fratello di questo, Giovanni, aveva sposato Giovanna da Montefeltro, figlia del duca Federico e sorella da parte di padre della nostra Gentile; ${ }^{5}$ non a caso quest'ultima, scomparso il marito, era a fianco di Giovanna durante l'assedio del Valentino alla rocca di Senigallia -giusto nell'anno della discutibile performance

1 Sarà sufficiente citare alcuni dei titoli di Rafael Beltrán Llavador (1988; 1990; 1997), che forse meglio di tutti conosce la questione.

2 Un agile raffronto in Alemany (1994) e, anche se datato, in Maravall (1964:15-31).

3 D'ora in avanti si citerà la versione di Ordóñez direttamente dall'originale, che titola per esteso Tragicocomedia di Calisto e Melibea novamente traducta de spagnolo in italiano idioma; nel farlo, ci si limita a distinguere $u$ da $v$, a convertire la cosiddetta s lunga, a sciogliere i compendi e ad adeguare maiuscole, accentazione e punteggiatura all'uso moderno. Quanto alla fonte del traduttore, rimando a Botta (2016).

4 Nell'edizione è definito «familiare de la sanctità di nostro signore Iulio papa secondo» (B1 v). Un compendio delle informazioni su questa edizione in Lampugnani (2015).

5 L'avvenimento teatrale è citato senza prove e in maniera ambigua da Alvisi (1978: 235). Per ogni informazione sulla Campofregoso rimando alla ricerca dettagliata di Paolini (2011).

SCRIPTA, Revista internacional de literatura i cultura medieval i moderna, núm. 14 / desembre 2019 / pp. 48 - 56 ISSN: 2340-4841 · doi:10.7203/SCRIPTA.14.16359 
Jacopo Gesiot. Ancora un plagio in Italia del Tirant lo Blanc: la lettera dedicatoria alla Tragicocomedia di Calisto e Melibea (1506)

celestinesca-, come vedremo un episodio tra gli altri piuttosto suggestivo per il tono della lettera di Ordóñez.

Se l'opera di Fernando de Rojas poteva rappresentare una novità per la nobildonna, d'altro canto riusciva forse più familiare e decifrabile il Tirant lo Blanc, che da un po' di anni circolava tra le corti grandi e piccole del nord Italia; si è infatti dimostrato altrove come per Mantova e Ferrara la conoscenza dell'opera di Martorell dovesse risultare pressoché obbligata, e, salvo il tentativo di traduzione di Niccolò da Correggio, realizzata in lingua originale (Gesiot 2018: 27-51). Non si è però riflettuto abbastanza intorno alla sua lettura nelle aule dei Montefeltro, un poco decentrate rispetto al triangolo filoiberico Ferrara-Mantova-Milano, ma naturalmente terminali per ogni novità, dato il legame con le principali casate padane. È così che, ricostruendo le vicende di una delle tre copie superstiti della princeps tirantiana, quella oggi conservata a New York dalla Hispanic Society of America, si giunge a una conclusione possibilmente rivelatoria: prima di passare per le mani di diversi bibliofili moderni, tra cui il barone Achille Seillière e Archer M. Huntington, l'incunabolo era stato conservato per più di un secolo presso la Biblioteca Universitaria Alessandrina, insieme al resto della collezione di libri a stampa (libraria nuova) dell'ultimo duca di Urbino, Francesco Maria II Della Rovere. ${ }^{6}$ Purtroppo, non siamo in grado di datare l'acquisizione della copia del Tirant, peraltro attestata, tra gli incunaboli cavallereschi tanto cari al duca (Vitaletti 1918): può trattarsi di un'entrata secentesca come di un'eredità del tempo dei Montefeltro. Non è neppure comprovabile la sua lettura da parte di Gentile da Campofregoso nel corso degli anni della vedovanza urbinate, eppure un'ipotesi di questo genere sarebbe perfettamente coerente con quanto sappiamo delle passioni iberiche di quel periodo.

I drammi in cui era piombata tutta la Penisola in seguito al contrasto per il trono napoletano, e che avevano compreso la morte del marito di Gentile, Agostino da Campofregoso, capitano pontificio nella guerra ai baroni filoangioini, stavano certo mettendo in crisi quelle certezze prolungate fino ad allora dalla politica italiana dell'equilibrio: sussulti di ordine morale ed esistenziale che spingono Alfonso Ordóñez a impiegare smaccatamente nell'apertura della dedicatoria una delle pagine più celebri del Decameron. È inimmaginabile che un lettore, anche minimamente istruito, non riconoscesse allora tra le righe il discorso di Pampinea ai giovani riuniti nella chiesa di Santa Maria Novella:

6 La prima ricostruzione della trafila si deve a Isidre Bonsoms i Sicart in occasione del discurso de recepción alla Reial Acadèmia de Bones Lleters, raccolto in Bonsoms y Sicart (1907). 
Illustrissima madonna, come io son certo che V.S. multissime volte habia inteso che a veruna persona fa ingiuria chi honestamente usa sua ragione. Natural cosa è adunque, de ciaschuno che nasce, sua vita quantunque può aiutare et conservare e quella difendere con ogni astutia et sollicitudine guardandosi da li adversi casi che in questa nostra humana vita con assai nostro danno vedemo ogni giorno succedere. $\mathrm{E}$ questo si concede tanto, che alchuna volta è già advenuto che, per guarda $<\mathrm{r}>$ la senza culpa alchuna si son commessi assai homicidii. Et concedendo ciò le leggi (nele solicitudini dele quali è il ben vivere de ogni mortale) quanto maggiormente, senza offesa d'alchuno, a noi et qualunque altro è honesto ala conservatione nostra prendere quelli congrui remedii che noi possiano (A2r)
Donne mie care, voi potete, così come io, molte volte avere udito che a niuna persona fa ingiuria chi onestamente usa la sua ragione. Natural ragione è, di ciascuno che ci nasce, la sua vita quanto può aiutare e conservare e difendere

E concedesi questo tanto, che alcuna volta è già addivenuto che, per guardar quella, senza colpa alcuna si sono uccisi degli uomini. E questo concedono le leggi, nelle sollecitudini delle quali è il ben vivere d'ogni mortale, quanto maggiormente, senza offesa d'alcuno, è a noi e a qualunque altro onesto alla conservazione della nostra vita prendere quegli rimedii che noi possiamo? (Branca 1987: 52)

Il tema della peste fiorentina, ben utile a descrivere le inquietudini del presente, implicava quello positivo dell'iniziativa femminile, incarnato dalla più grande e saggia tra le giovani fiorentine e, nella realtà, reso attuale dall'assenza temporanea -e no, come abbiamo visto per Gentile- dei mariti impegnati nelle campagne militari (Veglia 2008: 23-24). È così che, in questo punto, lo spagnolo innesta come per suggello una clausola dal prologo del Tirant, in modo da fissare l'indirizzo della sua iniziativa editoriale:

Et quanto sia la presente opera spechio et chiaro exempio e virtuoso [sic] doctrina a nostro ben vivere il nostro auctore per la presente opera chiaramente ce 'l dimostra, insegnandoci li aguati et inganni di coloro che poco amore ci portano, quali per ogni minimo loro utile non curano a chi di loro si fida con assai bisamo losenghevolmente ingannare, come nel processo di questi amanti compare (A2r)

In Martorell lo specchio era quello delle virtù guerriere del protagonista, qualità difficilmente attribuibili al più mite e urbano Calisto, sennonché a essere indicato nel nostro caso è un exemplum più prossimo all'ambito cortigiano che a quello militare, cionondimeno da perseguire con la stessa strenuità, particolarmente femminile. Qui poteva tranquillamente trovare applicazione l'avvertimento, reale o di comodo, da parte di Fernando de Rojas contro il «desordenato apetito» amoroso e, in particolare, le trame dei «malos e lisonjeros sirvientes» (Lobera, et al. 2000: 23). Di adulatori e consiglieri opportunisti, naturalmente ricercati e disprezzati in tutte le corti, arriverà di lì a poco a occuparsi Machiavelli, che dedica un capitolo intero a «quomodo adulatores sint fugiendi» (Inglese 1995: cap. 23); ma se la trattatistica di questo genere si rivolgeva anzitutto a un pubblico maschile, e si pensi anche alle «mosche» del Cortegiano, il tirocinio delle dame, sui cui pure potevano incombere obblighi amministrativi in assenza del signore, era allora meglio affidato agli esempi della letteratura di finzione. 
Al vertice della piramide sociale il motivo della trasparenza, con la scusa del concetto di fides amorosa, era legato sin dai tempi delle delizie medievali a quello della gerarchia feudale. Non per niente, come rivelano le sottolineature all'edizione italiana del Tirante (Venezia, Nicolini da Sabbio, 1538), ancora del pieno Cinquecento tra le principali preoccupazioni dei lettori ricorreva il tema della fedeltà e della sottomissione naturale alla struttura signorile (Martines 1997: 77-78); d'altronde, il romanzo catalano restitutiva un'immagine perfetta dell'antico ordine aristocratico-militare, sancito sul piano cosmologico dalla religione ufficiale e citato nel discorso del traduttore intorno all'influsso dei pianeti sull'arbitrio degli uomini. Ancora una volta, però, la fonte è Martorell:

Non per questo ali fraudulenti dala divina providentia fu e ne sarà lor perdonato, mostrandoce quanta iustitia sua bontà comparte e come fu in piacimento alo universal creatore che li cieli desseno influentia nel mondo e tenesseno dominio sopra la humana natura, donandoci diverse inclinationi di peccare et vitiosamente vivere, non per questo ne ha tolto il libero albitrio, che, se quello è ben governato, vivendo virtuosamente se può mitigare et vincere se usar volen de discreció (I 75) volemo discretione $(\mathrm{A} 3 r-\nu)$

La questione del libero arbitrio, che nel La Celestina è piuttosto legata al problema del philocaptio stregonesco, non si associa qui che vagamente all'amore, e quasi in maniera superficiale, perché lo spagnolo insiste:

\begin{abstract}
Onde io mosso da tal consideratione e vedendo la necessitate che tutti o la magior parte de questo presente tractato havemo, quale ci mostra apertamente via per la quale ci sapiamo guardare e difendere de l'inganni e losenghe de mali e tristi huomini (A3v)
\end{abstract}

A questo proposito, sulla funzione ammonitiva degli effetti d'amore, che ci si aspetterebbe da due esempi tanto dolorosi come quelli di Calisto e Melibea (e, in filigrana, di Tirant e Carmesina), prevale il pericolo esterno degli inganni, dei «mali e tristi huomini», una minaccia che non interessa solo il pubblico muliebre, ma, secondo quanto precisa Ordóñez, l’intera collettività, lui compreso («necessitate che tutti o la magior parte [...] havemo»). Il dibattito sull'amore carnale è così scansato, anche perché, tra i tanti contemporanei, se ne era già occupato un affine non proprio gradito alla destinataria, quel doge Battista da Campofregoso che, deposto dallo zio, marito di Gentile, aveva in seguito pubblicato l'Anteros, o Tractatus contra Amorem. ${ }^{7}$ A ogni modo, il tirocinio del capitano Tirant è più improntato alla prudenza cavalleresca, un paradigma politico e marziale per colui (o colei) che, non potendosi esimere dalla vita activa, diventava capace di schivare agonisticamente il proprio

\footnotetext{
7 Va tenuto presente che il trionfo della trattatistica amorosa nel Cinquecento era stato preceduto da una vivace offensiva teorica contro l'amor profano che, alla fine del secolo precedente, aveva coinvolto in particolare il Platina (Contra amores) e Pietro Edo (Antierotica).
} 
danno attraverso la lettura degli esempi passati (Pujol 2015: 124-126); anche di qui l'impiego nella seconda accezione della qualifica di tractato, scelta dal traduttore così come da Martorell, ma non da Rojas.

A questo punto Ordóñez si affida in tutto alla trama dello scrittore valenciano e arriva a innestare, con analoga funzione, una buona parte della dedicatoria del Tirant a un infante di Portogallo, questo sfruttando il topos letterario della falsa traduzione citato nella lettera a don Fernando d'Aviz. In realtà si tratta di un plagio al quadrato, dal momento che lo stesso Martorell aveva qui fatto ricorso al testo d'accompagnamento a Los dotze treballs d'Hèrcules, indirizzato da Enrique de Villena a Pere Pardo de la Casta, il che pone il nostro testo al termine di una discendenza di opere didattiche e morali (Pujol 2002: 20-22).

Fin a questo punto si è immaginata una certa consapevolezza positiva da parte del traduttore, e addirittura l'intenzione più o meno approfondita di risemantizzare il testo di partenza, tuttavia è altrettanto plausibile, anche se un po' rischioso, dato che il Tirant non era del tutto una primizia, che questi avesse optato per un raffazzonamento bello e buono. Così, una volta cercato di chiarire l'ispirazione di Ordóñez sulla scorta di un procedimento analogico, potremmo anche ammettere che non si intendesse più di tanto rendere esplicito questo meccanismo, al punto da autorizzare qualche accomodatura. In questa maniera il nostro plagiatore non esita a trasporre le sequenze del passaggio originale, nonché a operare alcuni cambiamenti più significativi:

Et anchora V.S., quale mossa da virtuoso desiderio, non per miei meriti, ma per sua virtù, s'è degnata volerme pregare dovesse io tradure la presente tragicocomedia intitulata di Calisto et Melibea de lingua castigliana in italiano idioma, aciò che V.S. insieme con questa degna patria dove questa opera non è divulgata se possa alegrare di tante e così degne sentientie et avisi che sotto colore de piacevoleze vi sonno.

Io adunque, vedendo che legitima obligatione di ubidire suoi preghi mi costringe, quali a me sonno stati acceptabili commandamenti, e per satisfare in parte al desiderio che di servir quella continovamente mi sporna meritamente me hanno obbligato a la executione di questa impresa, quantunque sia tenuto manifestare ogni opera virtuosa magiormente che per il presente tractato a quelli che lo legeranno, retenendo per sé le sententie necessarie et le lascivie lassando, grande utile ne venga; e como già sia considerata mia insufficientia e le curiali e familiari occupationi quali obstano ale adversità dela nobile fortuna che non dan riposo a miei pensieri, che di questo travaglio iustamente iscusar mi possa (A3v-A4r)
Per ço que la nació d'on yo só natural se'n puxa alegrar e molt ajudar per los tants e tan insignes actes com hi són (Dedicatoria 63)

Les quals pregàries són stades a mi molt acceptables manaments (Dedicatòria 61)

E jatsia considerada ma insufficiència e les curials e familiars occupacions qui obsten, e les adversitats de la noÿble fortuna qui no donen repòs a la mia pensa, de aquest treball justament excusar-me pogués (Dedicatòria 61) 
Al di là dei modi convenzionali della captazione, possiamo rilevare alcuni interventi più sensibili: in primo luogo la sostituzione del termine nació, di carattere marcatamente etnico-linguistico, con patria, più consono alla dimensione multinazionale delle corti italiane ed evocativo dell'impianto signorile e 'patriarcale' delle stesse; segue poi, oltre all'insistenza informativa (avisi), un richiamo al topos umanistico di ordine pedagogico-morale del miele (le piacevoleze) e del phármakon; un'attenzione infine, come già detto, alla nozione di tractato (quindi di sententie) coerente per l'appunto con il mandato del Tirant. Insomma, un'ispirazione che trascende quegli elementi licenziosi rilevati da più parti - più severo fra tutti sarà Giovan Battista Girladi (Villari 2002: 233 e 298) - e che secondo la prudenza del traduttore vanno necessariamente setacciati.

\section{E ancora:}

Ma confidandomi nel superno Idio donatore de tutti li beni, quale aiuta a li boni desiderii et suplisce ali diffecti di coloro che ben $\mathrm{fa}<\mathrm{r}>$ disiano e porta boni propositi spesse volte nele mente, et in V.S., quale per sua virtù comportarà li errori così in stilo como in ordine, se per me fusseno posti inadvertentemente ne la presente traductione, ché veramente non nego non vi se ne possa trovare, siando intrato in labirintho del quale me stesso a pena ne so trare. Per la qual cosa suplico humilmente V.S. voglia acetarla como de servitore affectionato, ché se falimenti alcuni vi sonno certamente, madonna, parte ne a colpa la dicta lingua castiliana, quale in alchune partite è impossibile posser ben tradure li vocaboli $[\ldots]$

Et aciò che di questa tragicocomedia lo primo auctore ne altri con epso non possa essere rimproperato, se errori alcuni li fusseno (como non dubito) V.S. voglia farli coreggere et amendarli, attribuendo la culpa di quelli a mio poco sapere et rudo ingegno et non al mancamento di mia voluntà desiderosa sempre di vostro servitio. Et aciò che li auctori per diffecto de miei falli non siano biasmati io solo voglio portarne il carico, come per me solo sia stata traduta al commando di V.S., a la cui gratia humilmente mi raccomando. Vale. (A4r-v)
Emperò, confiant en lo sobiran Bé, donador de tots los béns, qui ajuda als bons desigs supplint lo defalliment dels desijants e porta los bons propòsits a degudes fins, e vostra senyoria, qui per sa virtut comportarà los defalliments axí en stil com en orde, en lo present tractat per mi posats per inadvertència (Dedicatòria 61)

Supplicant vostra virtuosísima senyoria accepteu come de servidor affectat la present obra -car si defalliments alguns hi són, certament, senyor, n'és en part causa la dita lengua anglesa, de la qual en algunes partides és impossible poder bé girar los vocables (Dedicatòria 63)

E perquè en la present obra altri no puxa ésser increpat si defalliment algú trobat hi serà, yo, Johanot Martorell, cavaller, sols vull portar lo càrrech, e no altri ab mi, com per mi sols sia stada ventilada a servey de molt il lustre príncep e senyor rey spectant don Ferrando de Portugal la present obra (Dedicatòria 63)

In chiusura appaiono le formule consuete dell'excusatio (Gesiot 2018: 108-112), in primo luogo per la difficoltà di rendere in italiano una lingua straniera con vizi e virtù particolari, a cui possiamo noi aggiungere l'equivoco di certe connessioni etimologiche, tra le quali, più in alto, travaglio per treball e qui partite per partides. Interessante infine il tributo scrupoloso a «lo primo auctore», lungi dallimpulso piuttosto comune a confondere volutamente la genesi delle opere, in modo da far spiccare la mediazione di chi le traduceva. Per di più è tenuta in conto la questione, oggi spinosa (Botta 1995), della multipla autorialità della commedia (gli «altri con epso»), un particolare che 
Jacopo Gesiot. Ancora un plagio in Italia del Tirant lo Blanc: la lettera dedicatoria alla Tragicocomedia di Calisto e Melibea (1506)

Ordóñez riferisce fedelmente alle cc. B1 $v$-B2v dedicate da «Lo autore ad un suo amico» ( $\mathrm{El}$ autor a un su amigo»), seguite dal celebre acrostico spiegato altrimenti da Alonso de Proaza.

In questa parte conclusiva è stato quindi necessario ricomporre le fattezze confuse del capolavoro di Martorell, tuttavia, una volta smascherate, rimane il fatto di aver scoperto il primo esperimento superstite di un Tirantitaliano, nonché la controprova della sua concezione polivalente e, in entrambe le penisole, della sua fruizione quasi enciclopedica, anche al limite del centone.

\section{Bibliografia}

Alemany, R. (1994) «La mort de Tirant i el triomf d'Hipòlit o la crisi del món cavalleresc vista per un cavaller en crisì, dins Romero Muñoz, C. (ed.), La cultura catalana tra l'Umanesimo e il Barocco. Atti del Convegno dell'Associazione Italiana di Studi Catalani (Venezia, $24-27$ marzo 1992), Padova, Programma, pp. 13-26.

Alvisi, E. (1878) Cesare Borgia duca di Romagna, Imola, Ignazio Galeati e figlio.

Beltrán Llavador, R. (1988) «Paralelismos en los enamoramientos de Calisto y Tirant lo Blanc: los primeros síntomas del 'mal d'amor'», Celestinesca, 12/2, pp. 33-54.

—_.(1990) «Las «bodas sordas» en Tirant lo Blanc y la Celestina», Revista de filología española, 70, pp. $91-118$.

-(1997) «Eliseu (Tirant lo Blanc) ante el espejo de Lucrecia (la Celestina). Retrato de la doncella como cómplice fiel del amor secreto», dins Beltrán Llavador, R./Canet, J.L. (eds.), Cinco siglos de 'Celestina': aportaciones interpretativas, València, Universitat de València, pp. 15-42.

Bonsoms y Sicart, I. (1907) La edición principe del 'Tirant lo Blanch': cotejo de los tres ejemplares impresos en Valencia en 1490, unicos conocidos hoy en día, Barcelona, La Academia.

Botta, P. (1995) «Ancora sulla genesi e paternità de La Celestina», Cultura Neolatina, 55, pp. 269-283.

—. (2016) «La contaminación en N, la traducción italiana de La Celestina», Creneida, 4, pp. 107121.

Branca, V., ed. (1987) Giovanni Boccaccio, Decameron, Torino, Einaudi.

Gesiot, J. (2018) Romanzi tiranni. La prosa iberica di cavalleria nel primo Cinquecento padano, Roma, Aracne.

Hauf, A., ed. (2008) Joanot Martorell (Martí Joan de Galba), Tirant lo Blanch, València, Tirant lo Blanch.

Inglese, G., ed. (1995), Niccolò Machiavelli, Il principe, Torino, Einaudi.

Lampugnani, R. (2015) La prima traduzione italiana de 'La Celestina'. Primo commento linguistico e critico agli inizi del Cinquecento, Firenze, Olschki. 
Jacopo Gesiot. Ancora un plagio in Italia del Tirant lo Blanc: la lettera dedicatoria alla Tragicocomedia di Calisto e Melibea (1506)

Lobera, F.J., et. al., eds. (2000) Fernando de Rojas (y «antiguo autor»), La Celestina. Tragicomedia de Calisto y Melibea, Barcelona, Crítica.

Maravall, J.A. (1964) El mundo social de 'La Celestina', Madrid, Gredos.

Martines, V. (1997) El 'Tirant' poliglota. Estudi sobre el 'Tirant lo Blanch' a partir de les seues traduccions espanyola, italiana i francesa dels segles XVI-XVIII, Barcelona, Curial/PAM.

Paolini, D. (2011) «Madonna Gentile Feltria de Campofregoso, Alphonso Hordognez y la traducción italiana de La Celestina», eHumanista, 19, pp. 260-295.

Pujol, J. (2002) La memòria literaria de Joanot Martorell. Models i escriptura en el 'Tirant lo Blanc', Barcelona, Curial/PAM.

. (2015) Tirant lo Blanc, dins Badia, L. (ed.), Literatura medieval (iii). Segle xv, Història de la Literatura Catalana, Barcelona, Enciclopèdia Catalana/Barcino/Ajuntament de Barcelona, 2015, pp. 107161.

Veglia, M. (2008) «Il reggimento di Pampinea e l'esperienza giuridica del Decameron», dins Varallo, F. (ed.), In assenza del re: le reggenti dal XIV al XVII secolo (Piemonte ed Europa), Firenze, Olschki, pp. 1-34.

Villari, S, ed. (2002) Giovan Battista Giraldi Cinthio, Discorsi intorno al comporre, rivisti dall'autore nell'esemplare ferrarese Cl. I 90, Messina, Centro Interdipartimentale di Studi Umanistici.

Vitaletti, G. (1918) «La Biblioteca Comunale di Urbania e i suoi incunaboli», La Bibliofilia, 20/3, pp. 119-132. 